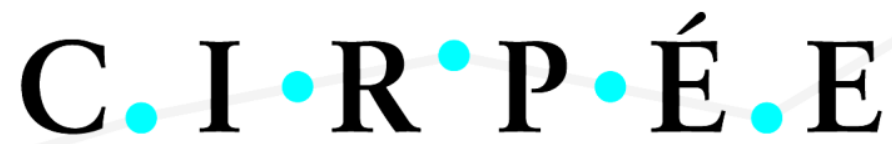

Centre Interuniversitaire sur le Risque, les Politiques Économiques et l'Emploi

Cahier de recherche/Working Paper 13-17

\section{Health Club Attendance, Expectations and Self-Control}

Jean-Denis Garon

Alix Masse

Pierre-Carl Michaud

Juillet/July 2013

Garon : ESG UQAM

Masse : ESG UQAM

Michaud : Corresponding author. ESG UQAM, RAND and CIRPÉE. Corresponding address : Département des sciences économiques, ESG UQAM, C.P. 8888, Succ. Centre-Ville, Montréal, QC, Canada H3C 3P8 michaud.pierre_carl@uqam.ca

We thank Jean-Yves Duclos, Marie-Louise Leroux and Pierre Lefebvre for their comments, participants at the Canadian Economic Association Meetings in Montreal 2013 and David Boisclair for editorial assistance. 


\begin{abstract}
:
Using a unique dataset on health club attendance from Quebec, we look at the relationship between actual and expected attendance and how these relate to measures of self-control. We find that a large majority of contract choices appear inconsistent if we do not take into account the commitment value of long-term contracts for attendees with self-control problems: $41 \%$ of members would be better off paying the fee for a single visit each time they go to the gym rather than signing a long-term contract. We then find that almost all members have made the right decision once we use subjectives expectations on the number of visits per week at the time of contract choice. We estimate that the median total cost is $\$ 229$ for those making a mistake. Next, we study how actual attendance following contract choice is related to measures of self-control. We find that reports of self-control problems at baseline are associated with low future attendance and that attendance decreases faster, in particular after New Year, for those expressing such problems. Quite interestingly, those expressing self-control problems do not expect at baseline to attend less often. We show that these results are consistent with a model where agents underestimate the severity of their self-control problems and estimate this degree of underestimation.
\end{abstract}

Keywords: Self-control, gym attendance, expectations, obesity

JEL Classification: D00, D12, D91 


\section{Introduction}

Physical activity is an important determinant of individual weight and health. The World Health Organization has recognized the importance to include it in strategies to fight the obesity epidemic, prevent disease and improve longevity (WHO, 2010). OECD countries have also engaged in designing preventive health policies in which physical activity holds a crucial role. ${ }^{1}$ Even when it does not entail a weight loss, the beneficial effects of exercising are manyfold. ${ }^{2}$

The design and effectiveness of policies targeting physical activity depend in large part on the determinants of individual behavior when it comes to do doing exercice. A significant fraction of vigorous physical activity is done in health clubs. The economics literature has recently begun analyzing the behavior of members at health clubs. This was in part motivated by anecdotal evidence that many members sign-off on long-term agreements while seldom attending after the first few months of the membership.

DellaVigna \& Malmendier (2006) collected data from three health clubs in New England. They found that the average price per visit for individuals with a membership was much higher than the price they would have paid without a membership. This could not be explained by pecuniary benefits associated with membership or by risk aversion. A key explanation was that members were too optimistic regarding future attendance at the time of choosing their membership. DellaVigna \& Malmendier (2006) collected additional information from a small sample of gym members in California to obtain information on attendance expectations and found that expectations appeared much higher than actual gym attendance. However, they could not survey the respondents of the health clubs for which they had data on attendance, which precluded them from analyzing the relationship between expectations and actual attendance at the individual level.

In two field experiments, Charness \& Gneezy (2009) analyzed how the frequency of gym attendance is influenced by monetary incentives. They found a large increase in participa-

\footnotetext{
${ }^{1}$ http://www.oecd.org/health/health-systems/theeconomicsofprevention.htm

${ }^{2}$ See Charness \& Gneezy (2009) for detailed literature.
} 
tion, entirely driven by self-reported low-attendance individuals. They also find evidence of habit formation. Their results were replicated by Acland \& Levy (2010), who also find that individuals over-predict future attendance. They interpret it as partial naiveté about one's self-control. Other studies, such as Babcock \& Hartman (2010), find that social pressures and herding may increase the frequency of physical activity.

To understand how expectations, actual visits and self-control are related, we present a model adapted from O'Donoghue \& Rabin (2001) where members potentially underestimate the extent of their self-control problems. To test predictions from the model, we constructed a unique dataset from a major health club organization with data on new members at 14 clubs in the Montreal area. In addition to data on the contract chosen in September 2011 and subsequent visits until May 2012 (9 months), we use reports from paper surveys conducted at the time of membership sign-up which asked questions on expectations of future attendance and on self-control problems. The unique feature of our data set is that it contains a self-assessed measure of motivation problems.

We first use these data to investigate whether contract choices are consistent with the expected price per visit, using both actual future visits (invoking rational expectations) and subjective expectations as revealed in the paper questionnaire. Second, we investigate how the discrepancies between actual and expected visits depend on the presence of selfcontrol problems. Finally, we investigate how the presence of self-control problems affects the evolution of attendance after sign-up.

Not taking into account the commitment value of long-term contracts for agents with self-control problems, we find that more than $40 \%$ of choices appear irrational when using actual visits, while more than $95 \%$ of choices are consistent once we rely on the expected number of visits at the time of signing the contract. We estimate that the median total cost is $\$ 229$ for those making a mistake. Furthermore, we find that expressed self-control problems correlate with actual visits to the gym but not with expected number of visits to the gym.Hence, members appear to underestimate the impact of their self-control problems.

In section 2, we lay out a model which allows us to derive predictions regarding the 
relationship between expectations, actual visits and self-control problems. In section 3, we present the data and methods used in the empirical analysis. Second 4 presents the results and section 5 concludes.

\section{Heath Club Contracting and Expectation Formation}

To understand how expectation formation, actual visits and self-control problems are related, we build a simple model in an environment which allows agents to potentially underestimate their degree of self-control. The model is adapted from O'Donoghue \& Rabin (2001).

We take an individual who lives for a large number of periods denoted by $t=0,1, \ldots$ One period represents an opportunity to exercise at a health club (for example, every two days). The timing of the problem is as follows: at $t=0$ the agent signs a membership contract with the health club. For all subsequent periods $t>0$, he sequentially decides whether or not to exercise. ${ }^{3}$

We denote a contract by a triple $(n, \theta(n), \gamma(n))$ where $n$ is the duration of the contract, most likely in days, $\gamma(n)$ is the fee that must be paid upon signing it, and $\theta(n)$ is a per-visit fee. Consistently with the facts that we have observed, we make the assumption that for any contract such that $n>1$ then $\gamma^{\prime}(n)>0$, but the daily price of membership falls with $n$, which requires that $n \gamma^{\prime}(n)<\gamma(n) \forall n$. For example, a twelve month contract could cost $\$ 30$ per month and a six month contract, $\$ 40$ per month. In the case of long-term contracts, $\theta$ is typically zero and $\gamma$ representing the present value of the (fixed) cost on the contract which does not depend on the number of visits.

Each visit to the gym generates a delayed (and implicitly discounted) payoff denoted by $h$, which stands for health benefits. We assume that $h$ is the same for all individuals. This assumption is not important to our results, and it will avoid redundancy with other utility parameters (discount factors). On the other hand, exercising at time $t$ is subject to

\footnotetext{
${ }^{3}$ Without loss of generality we make the assumption that rational individuals discount time geometrically at the unit rate $\delta=1$.
} 
an immediate random cost of effort, denoted by $e_{t}$. It is drawn from a time-invariant, IID distribution $F(e)$ with support $[0, \bar{e}]$. We assume, without loss of generality, that $F(e)$ is strictly increasing and twice continuously differentiable, with the associated density function $f(e)=F^{\prime}(e)$.

\subsection{Preferences and Optimization Problem}

We solve the model by backward induction. We first characterize the sequential decisions to exercise for three types of individuals: the rational agent; the sophisticated who have self-control problems at $t=0$ and who are conscious that these will persist in time; and the naive who are aware of their self-control problem at $t=0$ but who erroneously believe it to be temporary. We denote one's decision to exercise at $t$ by the binary choice function $g_{t}=\{0,1\}$, which depends on the realized shock $e_{t}$ and on all the exogenous parameters.

From an ex-ante perspective (i.e. $t=0)$, taking the contract $(n, \gamma(n), \theta(n))$ as given, an agent's expected utility for the duration of the contract is

$$
U_{0}=-\gamma(n)+E\left\{\sum_{t=1}^{n} g_{t}\left(e_{t}\right)\left[h-e_{t}-\theta(n)\right]\right\}
$$

where $E$ is an expectation operator on $F(e)$. However, from the perspective of any ex-post period $\tau>0$, the utility function, each period, of someone with a self-control problem becomes

$$
U_{\tau}=g_{\tau}\left(e_{\tau}\right)\left[\beta h-e_{\tau}-\theta(n)\right]
$$

Equation (1) is the agent's experience-utility, whereas (2) captures his decision-utility. The additional discount factor $\beta$ in (2) is allowed to differ across individuals. It represents the behavioral mistake that leads to time-inconsistent behavior. An individual with $\beta<1$ is considered to have a problem of self-control, which leads him to under-value the long-run health benefits $h$ as compared to the immediate cost of exercising $e_{t}{ }^{4}$

\footnotetext{
${ }^{4}$ Note that we have made the assumption that all individuals face the same function $F(e)$. We implicitly assume that all personal characteristics except self-control problems are contained in this function. More specifically, one can show that if the economy consisted of two different types of individuals, $i$ and
} 


\subsection{Objective (Actual) Number of Visits at $t>0$}

For $t>0$, an individual takes $(n, \gamma, \theta)$ as given. He observes his level of self-control $\beta$, and his realized cost of exerting effort $e_{t} \in[0, \bar{e}]$. He decides to visit the health club, a decision denoted by $g\left(e_{t}\right)=1$, if and only if

$$
-e_{t}-\theta+\beta h \geq 0
$$

Notice that the per-visit $\operatorname{cost} \theta$ reduces the likelihood that this condition will be satisfied. On the other hand, the up-front fee $\gamma$ is now sunk and does not appear in (3). Rearranging the last equation, we know that one exercises at $t$ if and only if $e_{t} \leq \beta h-\theta$. To guarantee an interior solution, we make the following assumption:

Assumption 1. $\beta h-\theta>0$ for all individuals.

The consequence of assumption 1 is that if the cost of effort is close to zero, then all individuals will decide to exercise. Although this does not drive our predictions, it generates interior solutions for the objective number of visits. From (3) we can derive the average number of visits of this individual from an ex-ante perspective, which we denote by $\operatorname{Ev}(\beta, \theta, n)$ :

$$
E v(\beta, \theta, n)=n F(\beta h-\theta) .
$$

From (4) one can see that, for any strictly increasing cumulative distribution function $F(e)$, the expected number of visits is increasing in $\beta$ and in $h$ and decreasing with respect to $\theta$.

Prediction 1. The objective number of visits is negatively correlated with reports of selfcontrol problems.

Proof. Suppose that at $t=0$ an individual reports his currently observed level of self-control $j$ respectively with $F^{i}(e)$ and $F^{j}(e)$ then we could rank individuals with respect to their fitness abilities. Here, assuming that $i$ is less fit (or that he generally faces higher costs of exercising) would be equivalent to assuming that $F^{i}(e)$, second-order stochastically dominates $F^{j}(e)$ on $[0, \bar{e}]$. We would thus obtain that, everything else being equal $v^{i}<v^{j}$. Without loss of generality, we keep working with a single type to isolate the effects of self-control problems and of sophistication on individual 
$\beta$. Then from (4)

$$
\frac{\partial E v(\beta, \theta, n)}{\partial \beta}=n h F^{\prime}(\beta h-\theta)>0 .
$$

\subsection{Subjective (Expected) Future Number of Visits at $t=0$}

We now focus on one's expectations about his future number of visits from an ex-ante perspective. Doing so allows us to introduce the notions of naiveté and of sophistication in our discussion. As shown before, any individual with $\beta<1$ is time-inconsistent and has problems of self-control.

Ex-ante, we assume that individuals can have erroneous beliefs about their future problems of self-control, which we denote by

$$
\hat{\beta}=(1-\alpha)+\alpha \beta \quad \in[\beta, 1] .
$$

The parameter $\alpha$ thus captures one's level of sophistication: an agent with $\alpha=1$ is fully aware, ex-ante, of his future motivation problems. On the other hand, $\alpha=0$ captures complete naiveté. We also allow for intermediate cases, where $0<\alpha<1$. Thus, at the time of choosing a contract, one's mistaken expectations about the number of visits to the health club are

$$
E \hat{v}(\hat{\beta}, \theta, n)=n F((1-\alpha)+\alpha \beta) h-\theta) \geq E v(\beta, \theta, n) .
$$

From (6) we can derive our second and third testable predictions:

Prediction 2. The expected number of visits will be larger than the actual number of visits when members are naive about their self-control problems.

Prediction 3. Reports of self-control problems correlate negatively with the subjective number of visits if and only if individuals are not perfectly naive. 
Proof. Deriving the subjective number of visits with respect to $\beta$ yields

$$
\frac{\partial \hat{v}(\hat{\beta}, \theta)}{\partial \beta}=\alpha F^{\prime}(\hat{\beta} h-\theta)
$$

which is strictly positive if and only if $\alpha>0$.

All three predictions are testable from data if we can observe expectations at the time of signing a contract, actual visits and self-reports of self-control problems. We next present the data we collected to test those predictions.

\section{Data}

We collected data from a large network of health clubs in Quebec. We focused on 14 clubs in the Montreal region. These clubs are centrally managed with harmonized pricing and contract menu. The amenities of each club are similar. We targeted all new contracts signed in September 2011. September is one of the busiest months (after January). New or renewing members choose a contract in September among the various options offered by the network. Contracts vary in terms of duration. Those with shorter durations are more costly per month. In the sample, the vast majority of contracts (over 95\%) are annual. At each visit, members swipe a card which automatically records attendance. These data are transmitted to the administrative office of the network. We obtained monthly attendance for each new member between September 2011 and May 2012.

We obtained 1171 valid membership records. The attendance file also contains information on date of birth, address, gender, status (new member or not), the type of membership, the price of the membership and the club in which the member has signed the contract. Members are allowed to attend any club in the network. These visits are recorded in the database irrespective of which club members go to. However, the vast majority of members attend the club where they signed the contract. We constructed the average number of visits per week as the ratio of the number of visits per month to the number of weeks in the month. We name this variable the objective number of visits per week. 
At the time of signing a contract, members were asked to fill in a paper questionnaire. We collected the paper questionnaires at each club and recorded the answers in a data file. The content of the questionnaire is varied. In particular it contains one question on the expected number of visits per week at the gym. A free form answer is recorded. We term this variable "subjective expected number of visits per week".

Members are also asked in the paper questionnaire about their problems with motivation. One question asks members whether they sometimes postpone training. An answer on a 5 point scale (never to often) is recorded. Another question asks whether members have difficulty maintaining the frequency of their training. An answer is recorded on the same 5 point scale. Answers to these two questions are highly correlated. We take an average of the scores on the 5 point scale. We then divide the respondents in two groups, those with a score of less than 2.5 and those with a score of more than 2.5. The second group is considered as having self-control problems while the first group is not.

In Table 1, we report descriptive statistics on the variables used in the analysis. Members are on average 33.9 years old and $56 \%$ are female. $84 \%$ of members who signed up in September 2011 had experience with health clubs while $80 \%$ were new members with this network. On average, members went 1.3 times per week at the club between September and May. But they expected to go almost 3 times per week at the time of signing their contract and filling the form. The average motivation index is 2.45 (before splitting in two groups) and $49.8 \%$ of members are classified as having self-control problems per our definition.

\section{[Insert Table 1 here]}

As can be seen from Table 1, not all members completed the form. Cases where no form was filled were rare. The number of valid records varied according to the question asked. For motivation questions, we recorded 650 valid answers. For the question on the expected number of visits, we recorded 825 valid answers. Non-response could be non-random. For those that did not fill a questionnaire, we have information on their actual number of visits, age, gender and club location. We estimated a logit model of the probability of missing 
information on these questions as a function of these covariates. Interestingly, none of the member level covariates were statistically significantly associated with missing information on the questionnaire. However, the amount of missing information varied across clubs with some clubs having much lower completion rates than others. Hence, in all analysis that involve regressions, we include club fixed effects.

\section{Results}

\subsection{Actual and Expected Visits}

We first look at the distribution of expected and actual visits per week to test Prediction 2. Figure 1 shows histograms. It is very clear that members overestimate the number of visits they will do on average per week. As seen in Table 1, the actual average number of visits is 1.3 while the average expected number of visits is 3.0 . This difference of 1.7 visits is statistically different from zero $(\mathrm{t}$-stat $=38.5)$. Hence, members are optimistic at the time of signing their contract. Less than $9 \%$ attend more than 3 times a week. The last panel in Figure 1 shows that underestimation is widespread and that very few actually overestimate the number of visits they will make on average.

[Insert Figure 1 here]

The fee based price is $\$ 14.95$ per visit. Hence, a natural thing to check is whether members actually go enough to the gym to make their membership worth it. The median monthly price of an annual membership is $\$ 39.10$. The price varies according to additional services, such as private training sessions that one might purchase. But the median price does not include additional benefits that a single visit would not provide. Hence, one needs to go to the gym more than 2.6 times per month, every month, to make the annual membership worthwhile.

In Figure 2, we plot the distribution of the cost per visit computed as the monthly price divided by the actual average number of visits per month and the expected number of visits 
per month. There is considerable variation in that cost with most of the variation coming from the number of visits rather than the price of the membership. Using the objective number of visits per month (actual), the average cost is $\$ 26.9$ per visit. Using $\$ 14.95$ as the cutoff for making the right decision, we obtain that only $59 \%$ of respondents have a cost per visit that is lower than the cost of a single pass.

We can compute the total cost of this mistake by taking the difference between the total cost of their membership over this period and subtracting the cost they would have incurred buying single passes. For those making a mistake over this period, the average error is $\$ 277$ while the median is $\$ 229$. The cost of making a mistake is large. For those making the right choice, given their actual number of visits, the average gain from buying an annual membership is $\$ 446.5$.

The bottom panel in Figure 2 reports the average cost per visit using the expected number of visits at the time of initial enrollment. Given the numbers we reported previously on the expected number of visits, it is not surprising to see that the bulk of the distribution is below the cutoff of $\$ 14.95$ for a single pass. In fact, $98 \%$ of members are making the right decision at the time of signing the contract, conditionally on their initial beliefs. But this optimism on the part of some members is costly ex post.

[Insert Figure 2 here]

\subsection{Determinants of Actual and Expected Visits}

This result raises an important question: who are these optimistic members? To answer it we look at the determinants of the average actual number of visits, $\log$ (objective) and the expected number of visits at the time of signing the contract, $\log ($ subjective). Finally, we also look at the determinants of the difference between the two by constructing an optimism indicator, $-\log$ (objective/subjective), which measures the $\%$ deviation between subjective and objective visits. We estimate a linear regression with age, gender, an indicator for self-control problems, two indicators for whether the respondent has experience with health clubs and is a new member of this network, and finally club fixed effects. The relationship 
with reports of self-control problems allows to test Predictions 1 and 3. Table 2 reports estimates.

[Insert Table 2 here]

The first column reports results for the objective number of visits. Those with selfcontrol problems have $20 \%$ lower attendance on average $(-0.205, \mathrm{t}=2.39)$. Hence reports of self-control are predictive of the actual number of visits which confirms prediction 1 . However, looking at the second column, we see that those with self-control problems do not expect to attend the gym less often than those without such problems $(-0.016, \mathrm{t}=0.81)$. Hence, Prediction 3 implies that agents are almost perfectly naive with respect to their selfcontrol problems. The last column confirms this. Those with self-control problems have an optimism index which is $17.9 \%$ higher than those without self-control problems.

\subsection{Recovering Sophistication}

From subjective and objective visits, we can recover the distribution of $\frac{\hat{\beta}_{i}}{\beta_{i}}$ where $i$ denotes the respondent. Let us assume that effort is $\log$ normally distributed such that $\log (e)$ is normally distributed with cdf $\Phi()$. Using this we have that $v_{i} / n=\Phi\left(\log \left(\beta_{i}\right)+\log \left(h_{i}\right)\right)$ while $\hat{v}_{i} / n=\Phi\left(\log \left(\hat{\beta}_{i}\right)+\log \left(h_{i}\right)\right)$. Using $\Phi^{-1}()$ to denote the inverse of the normal cdf, we then have a simple expression for a sophistication measure, $s_{i}$, given by

$$
s_{i}=\frac{\hat{\beta}_{i}}{\beta_{i}}=\exp \left(\Phi^{-1}\left(\hat{v}_{i} / n\right)-\Phi^{-1}\left(v_{i} / n\right)\right)
$$

In Figure 3, we show the distribution of $s_{i}$. The median estimate is 2.3 with a standard deviation of 2.2 confirming overall naiveté in our sample. Our respondents have on average a naive discount factor which is double their true discount factor. Less than $10 \%$ of respondents have a ratio under 1 .

[Insert Figure 3 here] 


\subsection{Predictive Value of Expected Visits and Self-Control}

One might be worried that the expected number of visits has no predictive value for the actual number of visits and this would explain why the expected number of visits does not correlate with self-control problems. To investigate this issue we estimate a poisson model, in panel, of the evolution of monthly visits as a function of age, gender, experience, new membership, self-control indicator and the expected number of visits per month at the time of signing the contract. We also include month fixed effects as attendance generally declines with time. We use both a pooled and a random effects poisson model (with gamma random effects). Table 3 reports estimation results.

[Insert Table 3 here]

The two specifications give similar results. The subjective number of visits per month is predictive of the number of actual visits per month. Expecting one additional visit per month is associated with a $16 \%$ increase in the actual number of visits. Given that the average number of visits per month is 5 , this represents roughly 0.8 additional visits. Hence, the self-reported expectations are highly predictive of actual visits. Since self-control problems did not correlate with the expected number of visits, one should not be surprised to find that even when controlling for the expected number of visits, those with self-control problems go less often to the gym. The magnitude of the effect in column 1 is similar to the one estimated in Table $2(-0.195, \mathrm{t}=2.55)$, confirming this suspicion.

An interesting question to ask is whether the effect of self-control problems is immediate at the start of the membership or only occurs after some time. To investigate this issue, we re-estimated a poisson model with gamma distributed random effects this time allowing for month fixed effects to be different between the two groups (those with self-control problems and those without). The estimated fixed effects relative to the number of visits in October of someone without self-control problems are plotted in Figure 4.

[Insert Figure 4 here] 
From October to December, the number of visits declines roughly at the same pace for both groups. During that period, the difference in attendance between the two groups is less than 10\%. There is an uptick in attendance in January for both groups. The gap between the two groups then becomes much larger starting in January. In May, members with self-control problems go to the gym $70 \%$ less often than when they started. The decline is less abrupt for those without self-control problems.

\section{Conclusion}

This paper uses new data on membership records from health clubs in Quebec to investigate the relationship between actual and expected gym attendance as well as the role played by self-control problems. We find that a large fraction of members is optimistic regarding their attendance at the time of signing their contract, and that those who are optimistic are more likely to also express problems with self-control. The cost of this over-optimism can be large, at the median close to $\$ 229$. Our findings are consistent with a model where members with self-control problems underestimate severely the future impact of their self-control problems on gym attendance.

That said, members who do not express self-control problems also suffer from optimism which could either mean that they suffer in general from optimism or that they are not aware of their self-control problems. Interestingly, our findings do not indicate that optimism is more prevalent among new members or members without experience with health clubs. This begs the question as to why members do not update their beliefs over time after witnessing that their expectations do not match what really happens in reality.

These results could have important implications for the design of membership plans. New members typically over-estimate the number of visits they plan to make to the gym. Although it might be profitable for networks to exploit this difference to lock-in members in expensive long-term contracts, the likelihood of renewing membership is unlikely to be high for members who realize that they actually did not go to the gym often. Hence, networks 
seeking to maximize long-term profits may actually care about renewing contracts instead of repeatedly having to go after new membership. Networks could potentially increase their renewal rate by first making members aware of overall patterns of behaviour in the population (optimism) and then targeting those who express self-control problems to try and increase their participation. Incentives such as reminders and text messages, etc, could potentially be useful to increase attendance, and ultimately renewals of memberships. The objectives of both governments and gym networks may be aligned in this case. 


\section{References}

(2010). Global recommendations on physical activity for health. World Health Organization.

Acland, J. \& Levy, M. (2010). Naiveté, projection bias and habit formation in gym attendance. Mimeo.

Babcock, P. \& Hartman, J. (2010). Exercising in herds: treatment size and status specific peer effects in a randomized exercise intervention. Mimeo.

Charness, G. \& Gneezy, U. (2009). Incentives to exercise. Econometrica, 77, 3.

DellaVigna, S. \& Malmendier, U. (2006). Paying not to go to the gym. American Economic Review, 96, 694-719.

O'Donoghue, T. \& Rabin, M. (2001). Choice and procrastination. The Quarterly Journal of Economics, 116, 121-160.

Strotz, R. H. (1955). Myopia and inconsistency in dynamic utlity maximization. Review of Economic Studies, 23, 165.

Uri Gneezy, S. M. \& Rey-Biel, P. (2011). Why and when incentives (don’t) work to modify behavior. Journal of Economic Perspectives, 4, 1-21. 


\begin{tabular}{lrrrrr}
\hline \hline Variable & Mean & Std. deviation & Min & Max & $N$ \\
\hline Age & 33.9 & 11.9 & 16 & 76 & 1165 \\
Female & 0.564 & 0.496 & 0 & 1 & 1171 \\
$\begin{array}{l}\text { Experience with } \\
\text { health clubs }\end{array}$ & 0.836 & 0.37 & 0 & 1 & 854 \\
$\begin{array}{l}\text { New member with } \\
\text { this health club }\end{array}$ & 0.781 & 0.414 & 0 & 1 & 1171 \\
$\begin{array}{l}\text { Objective visits/week } \\
\text { Subjective visits/week }\end{array}$ & 1.305 & 1.160 & 0 & 7.94 & 1171 \\
$\begin{array}{l}\text { Motivation index (1/5) } \\
\text { Self-control problems } \\
\text { (motivation index }>2.5)\end{array}$ & 2.997 & 0.749 & 1 & 7 & 8825 \\
\hline \hline
\end{tabular}

Table 1: Descriptive Statistics. Descriptive statistics on key variables used in the analysis. Please refer to text for variable definitions. 


\begin{tabular}{lccc}
\hline \hline Variable & Objective visits & Subjective visits & Optimism index \\
\hline Age & 0.001 & -0.003 & -0.006 \\
& $(0.3)$ & $(3.93)$ & $(1.46)$ \\
Female & -0.154 & -0.066 & 0.078 \\
& $(1.77)$ & $(3.31)$ & $(0.87)$ \\
Self-control problems & -0.205 & -0.016 & 0.179 \\
& $(2.39)$ & $(0.81)$ & $(2.03)$ \\
New member & -0.045 & -0.008 & 0.031 \\
& $(0.43)$ & $(0.35)$ & $(0.29)$ \\
Experience & -0.135 & 0.043 & 0.139 \\
& $(1.08)$ & $(1.50)$ & $(1.09)$ \\
Club fixed effects & Yes & Yes & Yes \\
$N$ & 608 & 596 & 583 \\
$R^{2}$ & 0.07 & 0.068 & 0.058 \\
\hline \hline
\end{tabular}

Table 2: Overall Attendance and Self-Control. Regression of log average objective visits, subjective visits and the log deviation between objective and subjective visits on controls as well as club fixed effects. T statistics are reported in parenthesis. 


\begin{tabular}{lcc}
\hline \hline Variable & Pooled poisson & $\begin{array}{c}\text { Random effects } \\
\text { poisson }\end{array}$ \\
\hline Age & 0.009 & 0.009 \\
Female & $(2.49)$ & $(2.34)$ \\
New member & -0.063 & -0.074 \\
& $(0.8)$ & $(0.87)$ \\
Experience & -0.049 & 0.038 \\
& $(0.53)$ & $(0.38)$ \\
Subjective visits & -0.096 & -0.048 \\
per month & $(0.96)$ & $(0.40)$ \\
Self-control & 0.160 & 0.170 \\
problems & $(3.31)$ & $(2.91)$ \\
Month fixed effects & -0.195 & -0.195 \\
Club fixed effects & $(2.55)$ & $(2.37)$ \\
log $(\alpha)$ & Yes & Yes \\
& Yes & Yes \\
$N$ & & -0.055 \\
log Likelihood & $-1.99 e 04$ & 0.96 \\
\hline
\end{tabular}

Table 3: Panel Attendance and Self-Control. Count models estimated on monthly data. First column reports coefficient of a pooled poisson model with clustered standard errors at the member level. Second column estimates a random effect poisson model where random effects are gamma distributed with variance alpha. This specification soundly rejects the hypothesis that alpha $=0($ Chi-square $=1.4 e 04)$. T-statistics are reported in parentheses. 

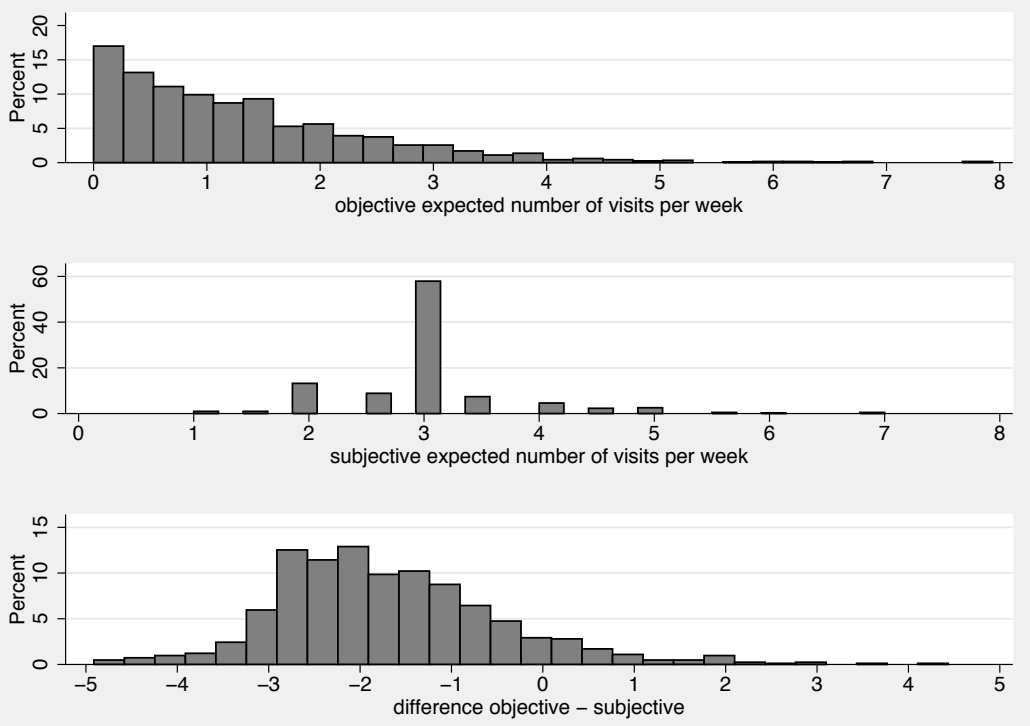

Figure 1: Objective and Subjective Number of Visits per Week 

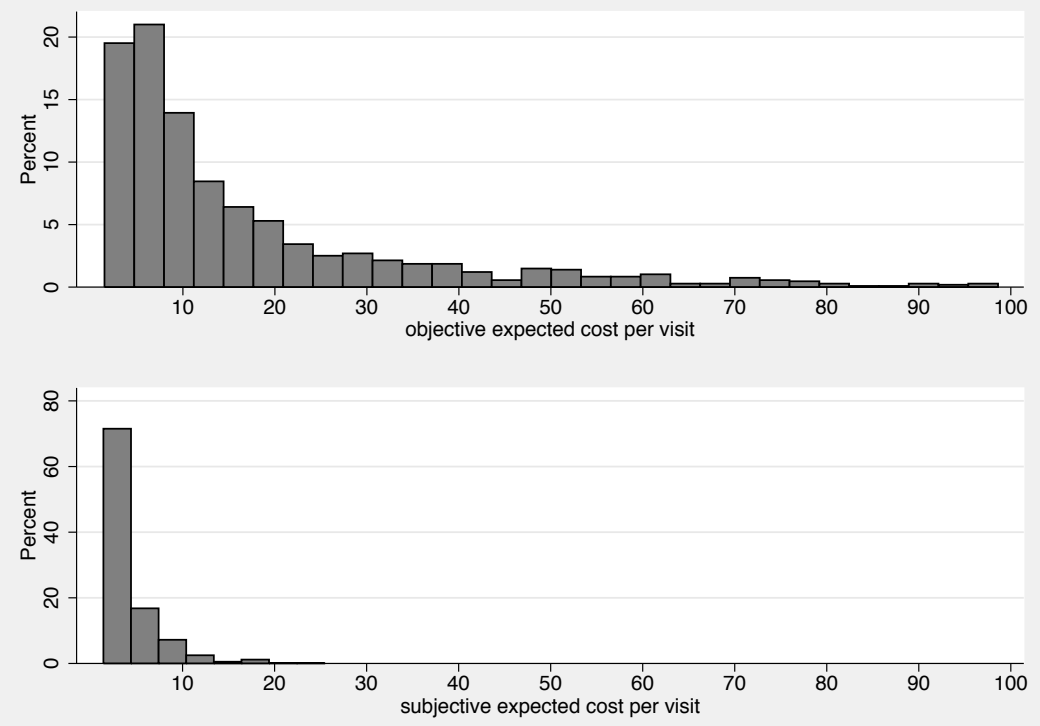

Figure 2: Objective and Subjective Expected Cost per Visit 


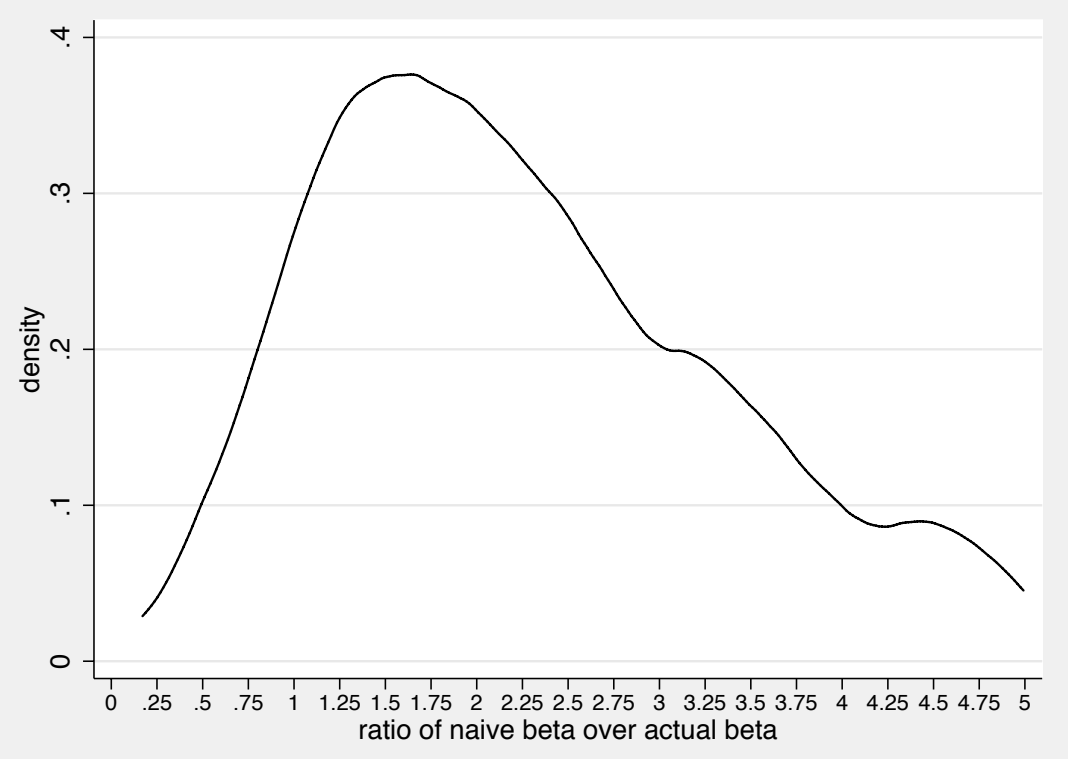

Figure 3: Degree of Sophistication Measured as the Ratio of $\hat{\beta}$ over $\beta$. 


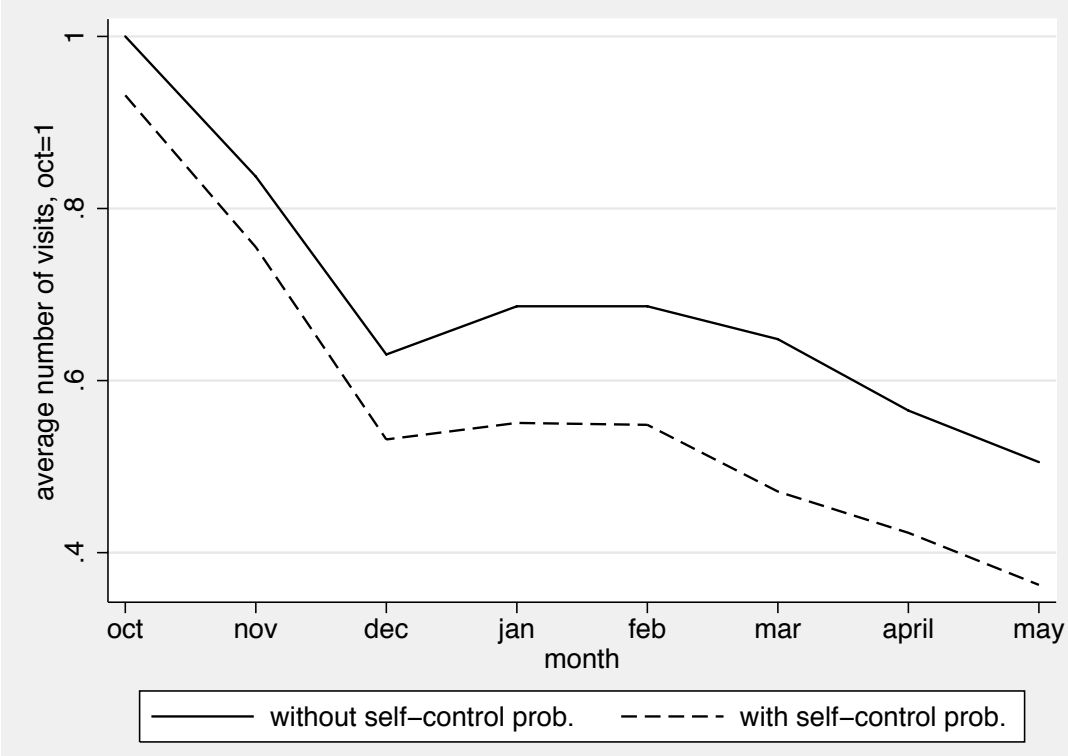

Figure 4: Average Number of Visits and Self-reported Self-Control Problems 